\title{
Distribution of long-period comets: comparison between simulations and observations
}

\author{
M. Fouchard ${ }^{1,2, \star}$, H. Rickman ${ }^{3,4}$, Ch. Froeschlé ${ }^{5}$, and G. B. Valsecchi ${ }^{6,7}$ \\ 1 University Lille 1, LAL, 59000 Lille, France \\ e-mail: marc. fouchard@univ-lille1.fr \\ 2 Institute de Mécanique Céleste et Calcul d'Éphémérides, 77 av. Denfert-Rochereau, 75014 Paris, France \\ 3 P.A.S. Space Research Center, Bartycka 18A, 00-716 Warszawa, Poland \\ ${ }^{4}$ Dept. of Physics and Astronomy, Uppsala University, Box 516, 75120 Uppsala, Sweden \\ 5 UNSA CNRS UMR 6202, Observatoire de la Côte d'Azur, Bd. de l'Observatoire, BP 4229, 06304 Nice Cedex 4, France \\ 6 IAPS-INAF, via Fosso del Cavaliere 100, 00133 Roma, Italy \\ 7 IFAC-CNR, via Madonna del Piano 10, 50019 Sesto Fiorentino (FI), Italy
}

Received 23 December 2016 / Accepted 29 April 2017

\begin{abstract}
Aims. This paper is devoted to a comparison between observations and simulations of the so-called Oort spike formed by the "new" observable long-period comets.

Methods. The synthetic distributions of observable comets come from the propagation of a huge sample of objects during the age of the solar system that were initially in a proto-Oort cloud, which was flattened around the ecliptic and had perihelia in the region of Uranus and Neptune. For the known new long-period comets, two samples were used, one that is assumed to be complete, and the comets of the other exclusively come from the Warsaw catalog of comets. The original orbital energy of the comets in this catalog is more reliable.

Results. Considering comets with a perihelion distance smaller than 4 AU, for which one of our samples of known comets can be assumed to be complete, the comparison shows small but significant differences in the orbital energy distribution and in the proportion of retrograde comets. When we extend the limiting perihelion distance to $10 \mathrm{AU}$, the observed samples are obviously strongly incomplete. The synthetic distribution shows that the number of observable comets per year and per perihelion distance unit is $\propto q^{1.09}$ for $q<4 \mathrm{AU}$ and $\propto q^{2.13}$ for $6<q<10 \mathrm{AU}$. The increase for $q>6 \mathrm{AU}$ comes from comets that were already within the Jupiter-Saturn barrier $(q<15 \mathrm{AU})$ at their previous perihelion passage (which we call creepers and Kaib and Quinn creepers), with original semi-major axes generally smaller than 20000 AU.

Conclusions. To explain the small but significant differences between our synthetic sample and the known comets for a perihelion distance smaller than $4 \mathrm{AU}$, different hypotheses are proposed: a still erroneous value of the original orbital energy in the observed sample, a higher density of low-mass stars in the actual solar neighborhood, a ninth planet, and obviously the initial population of objects from which the synthetic distributions are derived.
\end{abstract}

Key words. comets: general - celestial mechanics

\section{Introduction}

The aim of the present paper is to compare the distribution of orbital elements of observed long-period comets with an observable flux obtained from recent simulations made in Fouchard et al. (2017). These simulations were assumed to be as realistic as possible considering an Oort cloud formed from an extended scattered disk as the scenario proposed by Brasser \& Morbidelli (2013).

The question is which sample of observed long-period comets we have to consider for comparison. In studying longperiod comets, two constraints have to be taken into account: first, the sample should not contain any statistical bias, and second, the original orbital energy should be computed as accurately as possible. The latter point is specific to long-period comets since this original orbital energy is on one hand the key quantity that tells us from which part of the Oort cloud the comet is coming from, and on the other hand, this quantity is very difficult to

\footnotetext{
* Present address: Observatoire de Lille, 1 impasse de l'Observatoire, 59000 Lille, France.
}

compute because of its sensitivity to weak effects such as nongravitational forces.

Concerning the first point, because of the high increase in the discovery rate of comets since 1998, it is now possible to assemble a sample of comets that can be assumed to be complete. As regards the second point, the Warsaw catalog of comets (Królikowska 2014) is clearly the best catalog of quasi-parabolic comets because the original orbital energy has been computed in a very accurate way, using specific outgassing laws for each comet.

Unfortunately, the number of comets that satisfy both criteria is rather small. As a consequence, two samples of comets are considered: one for which the completeness of the sample can be assumed, and one for which the original orbital energy has been computed accurately.

These two samples of observed long-period comets are described in Sect. 2. Section 3 is devoted to a brief description of our simulations, which have been used to construct different samples of synthetic observable comets. Comparisons between simulations and observations are made in Sect. 4 using an 


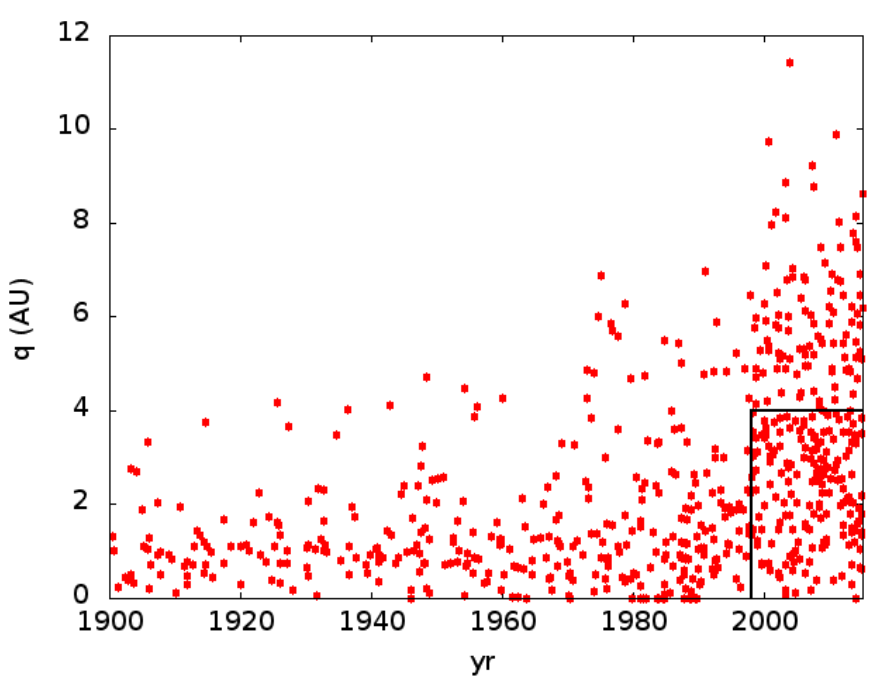

Fig. 1. Osculating perihelion distance versus time of the perihelion passage for all discovered long-period comets from 1900.

observable limit at $4 \mathrm{AU}$ (Sect. 4.1) and at $10 \mathrm{AU}$ (Sect. 4.2). Then the conclusions are given in Sect. 5.

\section{Observed samples}

We here focus on the flux of new long-period comets, that is, comets whose original semi-major axis is greater than $10000 \mathrm{AU}$. By original semi-major axis we mean the barycentric semi-major axis of the comet before it has been affected by planetary perturbations or non-gravitational forces. Comets are usually called new long-period comets when it is thought that they are coming within the Jupiter-Saturn region for the first time.

However, it appears both from observations (Dybczyński \& Kròlikowska 2011, 2015) and from numerical experiments (Fouchard et al. 2014, 2017) that this is not the case, and that a large proportion of new comets have been in the region of Jupiter and Saturn at their previous perihelion passage. This proportion depends on the threshold used to define the JupiterSaturn barrier. Using $15 \mathrm{AU}$ in both cases yields a proportion of "fake" new comets in the observations sample of around $50 \%$ (51\% for Dybczyński \& Królikowska 2011) and (49\% for Dybczyński \& Królikowska 2015), and from the simulations, this proportion is about $61 \%$ in Fouchard et al. (2014) and 55\% in Fouchard et al. (2017). We still refer for historical reason to these comets as the new long-period comets.

For the known comets it is not a simple task to recover the original orbital energy and thus to select which comets are new. We discuss this point later.

Figure 1 shows the osculating perihelion distance versus the time of perihelion passage for each discovered new long-period comet since 1900 with a total absolute magnitude lower than 11 . The total magnitude used is the one given by the JPL/Horizon system (Giorgini et al. 1996). When no magnitude is given, which generally occurs for some rather old comets, we have considered that the total magnitude was smaller than 11 . Five recent comets (C/2000 S5, C/2002 Q3, C/2004 H1, C/2004 YJ35, and C/2006 M2), which were discovered after 1998, had no magnitude given in the JPL/Horizon system. Using the Minor Planet Electronic Circular, we made a rough estimate of the total visual absolute magnitude $H$ according to

$H=m-5 \log \Delta-2.5 n \log r$,

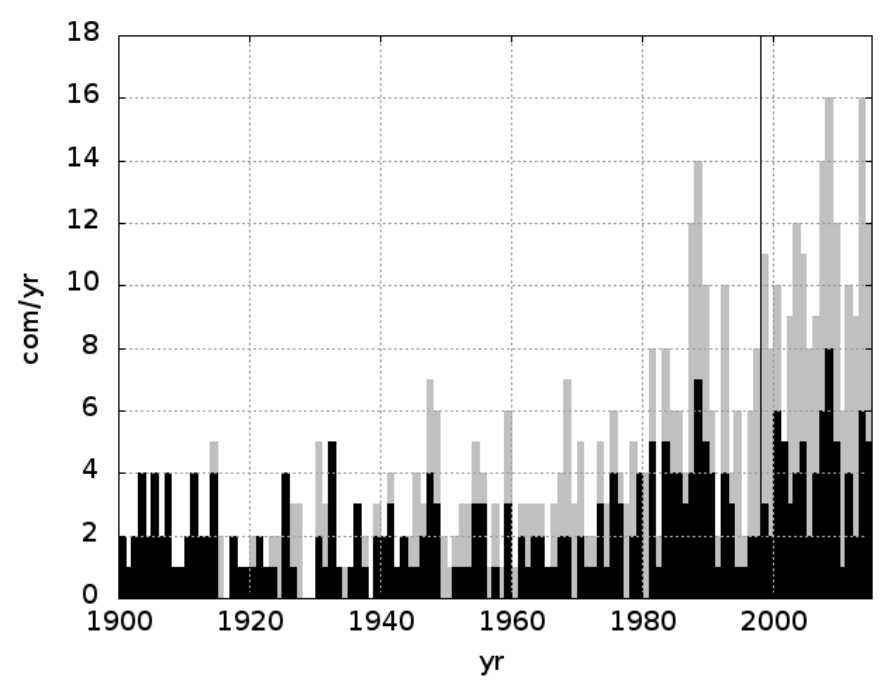

Fig. 2. Number of discovered long-period comets per year with a perihelion distance smaller than $4 \mathrm{AU}$ and a total absolute magnitude smaller than 11 each year since 1900. The gray histogram corresponds to all the comets in the Horizon/JPL data base, whereas the black historgram shows only the new comets in the JPL sample (see text for details).

where $m$ is the total magnitude, $\Delta$ and $r$ are the geocentric and heliocentric distance (in $\mathrm{AU}$ ), and $n$ is the photometric index ${ }^{1}$. We obtained 10, 18, 13.9, 12.4, and 10 for the five comets. Two comets (C/1975 E1 and C/1996 R3) had a nuclear absolute magnitude but no total absolute magnitude. For both of them the nuclear magnitude was close to or below our threshold of 11 for the total magnitude. Consequently, we consider that for these two comets the total absolute magnitude was smaller than 11. We clarify that it is beyond the scope of the present paper to investigate the magnitude of the comets. According to Fernández \& Sosa (2012), the magnitude distribution of longperiod comets reaches a ceiling at about 12 , and this ceiling is not caused by selection effects. Recent surveys such as LINEAR have a higher detection limit than this. However, in surveys conducted before this, the sample of comets with magnitudes smaller than 11 might be incomplete.

Figure 1 shows the perihelion distance versus time of the perihelion passage for all discovered long-period comets since 1900. A clear jump in the discovery efficiency is observed in 1998, which corresponds to the beginning of the LINEAR program. Despite the beginning of other surveys such as the Catalina Sky Survey (overcoming LINEAR discoveries in 2005), PAN-Starrs (2012), and the improvement of LINEAR instruments (2014), no jump in the discovery rate is clearly observed later for comets with a total magnitude lower than 11 and a perihelion distance smaller than $4 \mathrm{AU}$.

Figure 2 shows the number of discovered long-period comets with a perihelion distance smaller than $4 \mathrm{AU}$ and a total absolute magnitude smaller than 11 every year since 1900. The figure also shows the number of discovered new comets in the JPL sample (see below for how this sample is obtained). The jump in the discovery rate with the beginning of the LINEAR survey is well observed. It is unclear whether a global increase caused by an overall improvement of the comet discovery with time is present later, or if we only observe some statistical fluctuation in the cometary flux. Nevertheless, we can stress that the number of discovered comets with perihelion distances smaller than

\footnotetext{
1 For simplicity, we assumed $n=4$, which gives the standard total
} magnitude $H_{10}$. 
$4 \mathrm{AU}$ and a total absolute magnitude smaller than 11 is nearly complete.

This sample was used to build a first set of observed new comets and is assumed to be nearly complete, thus unbiased. We still have to compute the original orbital energy for each comet, that is, the comet barycentric orbital energy before it was affected by the planets and non-gravitational forces.

To do so, the JPL-Horizon system (Giorgini et al. 1996) was used to integrate each comet backward until a heliocentric distance greater than $150 \mathrm{AU}$ was reached. The integration model is based on DE406 using the ICRF/J2000 reference frame, and the ecliptic and mean equinox of reference epoch for the barycentric coordinates. The non-gravitational forces are included. At this distance from the Sun, we assume that the orbit is barycentric, which allows us to compute the original orbital energy. Todorovic-Juchnicwicz (1981) proposed a distance of between $150 \mathrm{AU}$ and $200 \mathrm{AU}$. For our comets the difference between the barycentric orbital energy computed at $150 \mathrm{AU}$ and $200 \mathrm{AU}$ was never greater than $10^{-8} \mathrm{AU}^{-1}$. In the following, by orbital energy we mean the quantity $z=-1 / a$, where $a$ is the semi-major axis (with a negative sign in the case of hyperbolic orbits).

The first set of observable comets, called the JPL sample, is then the set of comets with a total magnitude smaller than 11, a perihelion distance smaller than $4 \mathrm{AU}$, a perihelion passage between January 1, 1998, and March, 27, 2015, and an original orbital energy $z_{\text {orig }}$ computed through the JPL-Horizon system, such that $z_{\text {orig }}>-10^{-4} \mathrm{AU}^{-1}$, that is, $a_{\text {orig }}>10000 \mathrm{AU}$ or $a_{\text {orig }}<0$. This sample contains 63 comets, 9 of which have a hyperbolic orbit.

It is known that non-gravitational forces may significantly affect the original orbital energy of long-period comets (Królikowska 2006), but these forces are still difficult to estimate for long-period comets because they have been observed only once at their perihelion. Consequently, when available, the original orbital energy obtained from the Warsaw Catalog of cometary orbits (Królikowska 2014) was considered instead of the energy computed from the JPL-Horizon system. This choice is made because the modeling of non-gravitational forces is more accurate in the Krolikowska catalog, mainly because all observations are used and weighted to determine the parameters involved in these forces. Then, as was shown in Królikowska (2006), this significantly affects the original orbital energy.

Of the 63 comets of the JPL sample, 36 are also present in the Warsaw catalog. Of these, 2 comets (C/1999 H3 LINEAR and C/2008 A1 McNaught) cannot be considered as new because their semi-major axes are smaller than $10000 \mathrm{AU}$, while C/2007 Q1 Garradd, which should be considered as old according to the JPL Horizon system, is reclassified as new in the Warsaw cata$\log$. Consequently, our second sample is the JPL $\mathrm{WC}_{\mathrm{WC}}$ sample, containing 62 comets, 35 of which have the original orbital energy taken from the Warsaw catalog.

From the JPL $\mathrm{WC}_{\mathrm{W}}$ sample of comets, which is assumed to be complete, we can deduce a mean flux of comets with a total magnitude lower than 11 and a perihelion distance smaller than $4 \mathrm{AU}$ of 3.6 comet/year. This value is rather consistent with the value obtained in Francis (2005) from LINEAR observations alone, and also with the simulations made in Fouchard et al. (2017), where in each case a flux of about $4{\text { comet } / y e a r s^{-1}}$ for a perihelion distance smaller than $5 \mathrm{AU}$ and a total magnitude lower than 11 was estimated. This does obviously not prove that our $\mathrm{JPL}_{\mathrm{WC}}$ sample is complete, but at least it shows that we are near to completeness.

The JPL ${ }_{W C}$ sample is clearly better than the JPL sample, as we show later, but 27 comets were not in the Warsaw catalog,

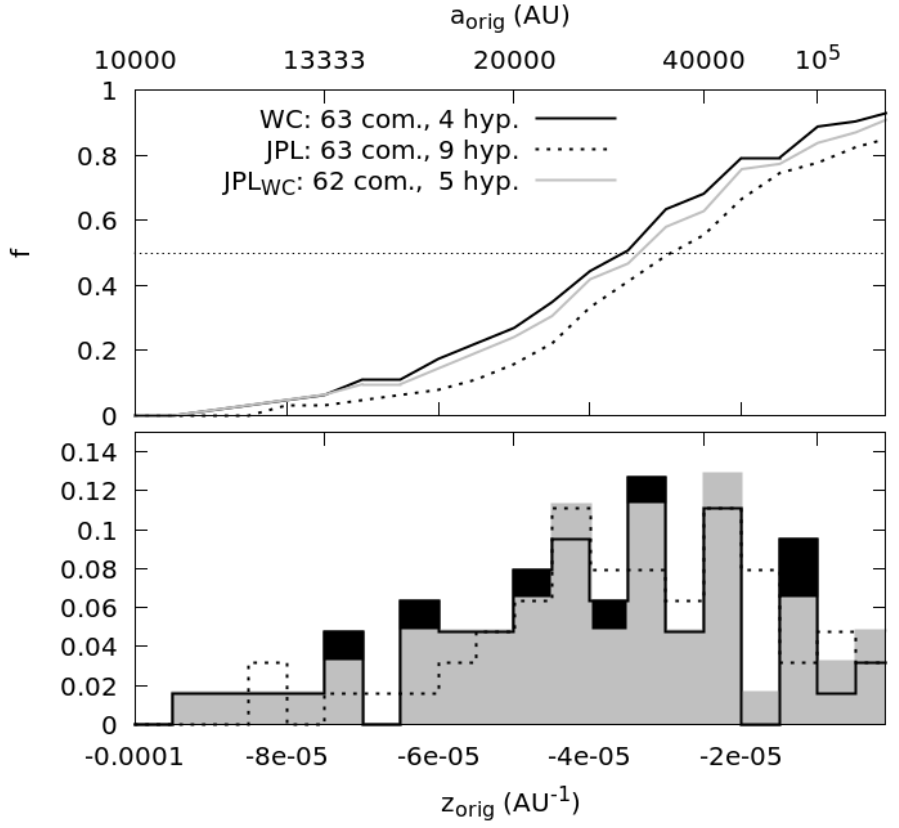

Fig. 3. Distribution of the original orbital energy $z_{\text {orig }}$ for the three samples of observable comets we used. The top plot shows the cumulative distributions, and the bottom plot shows the repartition functions. All numbers are normalized to the total number of comets in each sample. The legend in the top left corner indicates the color-coding we used, the number of comets, and the number of hyperbolic comets in each sample.

which means that $z_{\text {orig }}$ is not properly computed for them. Therefore, we used a third sample that we call the WC sample. It contains comets that are in the Warsaw catalog and whose perihelion distance is smaller than $4 \mathrm{AU}$, the perihelion passage was after January 1, 1950, and the original orbital energy is such that $z_{\text {orig }}<10^{-4} \mathrm{AU}^{-1}$. No filtering was made on the total magnitude because it is not expected to introduce biases in the distribution of orbital elements (so far, no such biases have been identified). However, this sample cannot be considered complete because before 1998 the detection of comets was clearly not complete for perihelion distances smaller than $4 \mathrm{AU}$. We could have set a threshold for the total magnitude smaller than 11 or reduced the perihelion distance, but it would have reduced the number of comets in the sample. In addition, we already have sample JPL and $\mathrm{JPL}_{\mathrm{WC}}$, which are assumed to be complete.

The Oort spike, that is, the distribution of the original orbital energy $z_{\text {orig }}$ for the three samples, is shown in Fig. 3. The top plot shows the cumulative distributions and the bottom plot the density distribution. In both cases the number of comets is normalized to the total number of comets in the sample. We note that a proper modeling of non-gravitational forces induces a reduction of the deduced original orbital energy. This has been observed in Królikowska (2006). The reduction is evident between the JPL and the JPL ${ }_{W C}$ samples. However, even between the JPL ${ }_{W C}$ and the WC samples, an overall reduction is observed. The median value of the original semi-major axis for the JPL sample is $33400 \mathrm{AU}$, whereas it is $30900 \mathrm{AU}$ for the JPLWC sample and $28200 \mathrm{AU}$ for the WC sample.

From now on, we consider only the JPL $\mathrm{WC}_{\mathrm{WC}}$, which is assumed to be complete, and the WC samples, which are incomplete, but have a more reliable $z_{\text {orig }}$. 


\section{Model and simulations}

Fouchard et al. (2017) presented a long-term propagation of an initial set of $10^{7}$ comets. Initially, the comets are in a kind of extended scattered disk with a semi-major axis of between $1100 \mathrm{AU}$ and $50000 \mathrm{AU}$, a perihelion distance between $15 \mathrm{AU}$ and $32 \mathrm{AU}$, that is, in the region of Uranus-Neptune, and an ecliptical inclination lower than $20^{\circ}$. The initial orbital energy, perihelion distance, inclination, argument of perihelion, longitude of the ascending node, and mean anomaly are all randomly chosen with uniform distributions.

Each comet is propagated taking planetary perturbations, galactic tides and stellar encounters into account. For the galactic tides the parameters are the usual ones taken from Levison et al. (2001), and the stellar encounters sequences are computed as is described in Rickman et al. (2008). For each comet the propagation stops if the comet reaches a heliocentric distance greater than $400000 \mathrm{AU}$, or impacts the Sun or any giant planet, or has a semi-major axis smaller than $100 \mathrm{AU}$.

Five different records of the surviving comets are performed after a propagation lasting four to five billion years. Each record corresponds to a possible final Oort cloud. Between each recording time, a complete revolution of the solar system around the Galactic center is performed so that each recording snapshot of the Oort cloud can be considered uncorrelated from the others.

From each final Oort cloud an additional propagation, called relaxing period, is performed during $30 \mathrm{Myr}$, during which all shower-making star passages are removed. This precaution is taken because it is unlikely that we are currently observing a comet shower (Wiegert \& Tremaine 1999).

At the end of the relaxing period, the position of the galactic center corresponds to the present galactic center. This second precaution comes from the fact that the effect of the radial component of the tides depends on the position of the galactic center with respect to the ecliptic, and this may affect the flux of observable comets (Levison et al. 2006).

Then, the first perihelion passage after this relaxing period is considered for each comet of our synthetic sample of observable new comets. We then gather the data obtained from the five final Oort clouds considering that the results correspond to an initial population of 50 million comets. For more details about the model and simulations, we refer to Fouchard et al. (2017).

In Sect. 4 we consider two different thresholds of the maximum heliocentric distance for a comet to be observable: $4 \mathrm{AU}$ and $10 \mathrm{AU}$. These comets are shared in four different classes according to the way they became observable (Fouchard et al. 2014). These classes are

- the jumpers, consisting of comets for which the perihelion distance passed from values higher than $15 \mathrm{AU}$ into the observable region during the last orbital period before being observable;

- the creepers, consisting of comets whose perihelion distance was smaller than $15 \mathrm{AU}$ at the perihelion passage that occurred one orbital period before the observability;

- the Kaib and Quinn jumpers, consisting of jumpers for which the quantity $z=-1 / a$ (proportional to the orbital energy) increased by more than $10^{-4} \mathrm{AU}^{-1}$ at the perihelion passage preceding the observability;

- and the Kaib and Quinn creepers, the same as Kaib and Quinn jumpers, but for creepers.

The term Kaib and Quinn comes from Kaib \& Quinn (2009), who highlighted the process in question for the first time in the framework of Oort cloud dynamics.

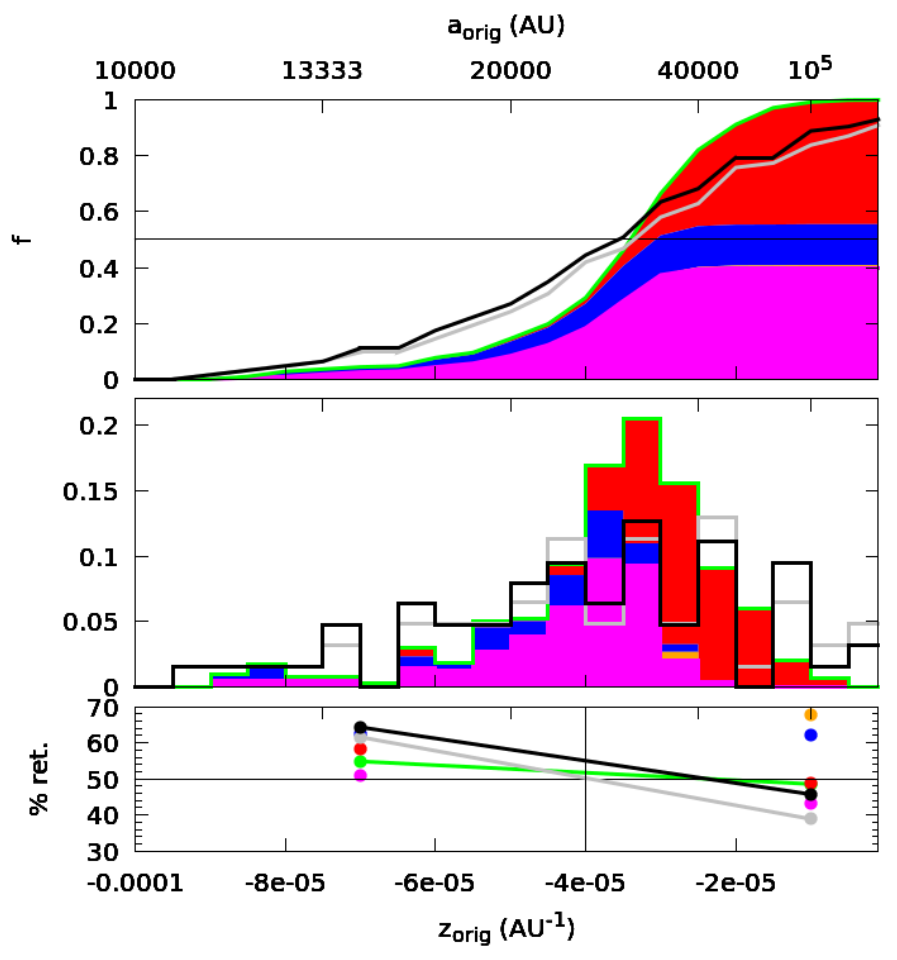

Fig. 4. Top panel: cumulative distribution of the original orbital energy for the observable new comets obtained from the simulations (green line), for the JPL $\mathrm{WC}_{\mathrm{WC}}$ sample (gray line), and for the WC sample (black line). For the simulations, the color width is proportional to the numbers of comets in each class. Red, blue, orange, and magenta correspond to the jumpers, creepers, KQ-jumpers, and KQ-creepers, respectively. Middle panel: number of comets per bin of constant orbital energy width normalized to the total number of comets for each sample. Each been is colored according to the proportion of comets in each class. Bottom panel: proportion of retrograde orbits for a semi-major axis smaller than $25000 \mathrm{AU}$ and a semi-major axis greater than $25000 \mathrm{AU}$. The color-coding for the middle and bottom panel is the same as for the top panel.

Ten different simulations were performed using different sequences of stellar encounters. These simulations are the same as those used in Fouchard et al. (2017). We illustrate most of the result using the same stellar sequence that is assumed to be a median case. When necessary, the other stellar sequences are used to evaluate the range of the results.

\section{Simulations versus observations}

\subsection{Observable comets with $q<4 \mathrm{AU}$}

\subsubsection{Orbital energy and inclination}

For the simulation, a comet is observable if it is located closer than 4 AU from the Sun after its first perihelion passage after the relaxing period. In order to take the probability for the comet to be observed into account, we transposed each observable comet in the simulation by a number of comets per million year. To do so, we applied the same rule as in Fouchard et al. (2017), where each observable comet in the simulation was weighted by $10^{6} / P_{\text {orig }}$, where $P_{\text {orig }}$ is the original orbital period of the comet (counted in years).

Figure 4 shows the distribution of the original orbital energy for the new observable comets obtained from our simulations and for the JPL $\mathrm{WC}_{\mathrm{C}}$ and WC samples. The top panel shows 
the cumulative distribution, whereas the middle panel shows the density distribution. In each case the numbers are normalized to the total number of comets considered. The bottom panel shows the proportion of retrograde orbits for two regions of the Oort spike: one with a semi-major axis smaller than $25000 \mathrm{AU}$, and one with a greater semi-major axis. This critical semi-major axis is very close to the critical value below which the tides are unable to produce jumper comets on their own (Fouchard et al. 2011).

The Oort spike obtained from our simulations is narrower than the Oort spike obtained from any of the two observed samples. However, for the synthetic sample, the median of the semimajor axis is equal to $29300 \mathrm{AU}$. This value is consistent with the medians obtained from the JPL $\mathrm{WC}_{\mathrm{WC}}$ and WC samples. Considering the first quartile, a significant discrepancy is observed, with a higher value for the synthetic sample (23800 AU) than for the two observed samples (20800 AU and $18700 \mathrm{AU}$ for the $\mathrm{JPL}_{\mathrm{WC}}$ and WC samples, respectively).

For all three samples the trend of the proportion of retrograde comets is the same: a preference for retrograde comets for comets with $a_{\text {orig }}<25000 \mathrm{AU}$, and either no preference or a preference for prograde orbits otherwise. This global trend is well explained in Fouchard et al. (2017) and is caused by the fact that above $25000 \mathrm{AU}$ we find mainly jumper comets that do not have any preference between prograde and retrograde, whereas below $25000 \mathrm{AU}$, we have mainly creepers and KQ-creepers that have a preference for retrograde orbits. The main difference between the three samples is observed between the JPL $\mathrm{WC}_{\mathrm{W}}$ and the WC sample for comets with $a_{\text {orig }}>25000 \mathrm{AU}$, where the preference for prograde orbits is strong $(62 \%)$ for the JPL $L_{W C}$ sample, whereas it is only $54 \%$ for the WC model. For the simulation sample the preference for prograde orbit is even smaller $(52 \%)$. This discrepancy may be explained by the fact that about half of the comets in the JPL $\mathrm{WC}_{\mathrm{C}}$ sample are not in the Warsaw catalog. Hence, for these comets, $a_{\text {orig }}$ is poorly defined. As we noted before, a more careful calculus of $a_{\text {orig }}$ usually decreases its value. Then we most likely have some comets in the JPL $L_{W C}$ sample for which $a_{\text {orig }}$ is erroneously greater than $25000 \mathrm{AU}$. This is enough for three of them to be on a prograde orbit to show proportions of retrograde comets in the two parts of the Oort spike that converge toward our simulation sample.

Considering the ten simulations using different stellar sequences, we can evaluate how sensitive our results are to the stellar sequences we used. Figure 5 shows the range of the cumulative distribution of the original orbital energy for the new comets and the range of the proportion of retrograde orbits for the two regions of the Oort spike defined before.

The first quartile and the median of $a_{\text {orig }}$ for the comets in the Oort spike range in [21400-23 $800 \mathrm{AU}$ ] and [28 100$29900 \mathrm{AU}$, respectively. All the medians are well between the value obtained for the WC and the JPL $\mathrm{WC}_{\mathrm{WC}}$ samples, whereas all the quartiles are greater than what was obtained for the two observed samples. The discrepancy on the first quartile is thus confirmed.

For the proportion of retrograde comets with $a_{\text {orig }}<$ $25000 \mathrm{AU}$, we note that the range obtained from the ten synthetic samples is very large. However, the results obtained with the observed samples are within this range. For comets with $a_{\text {orig }}>25000 \mathrm{AU}$, the simulation samples span the equilibrium between retrograde and prograde orbits. No synthetic sample has a preference for prograde comets greater than $52 \%$, however, whereas it is about $54 \%$ for the WC and $62 \%$ for the JPL $L_{W C}$ sample.

Consequently, some discrepancies exist on the distributions of the original orbital energy and on the proportion of retrograde

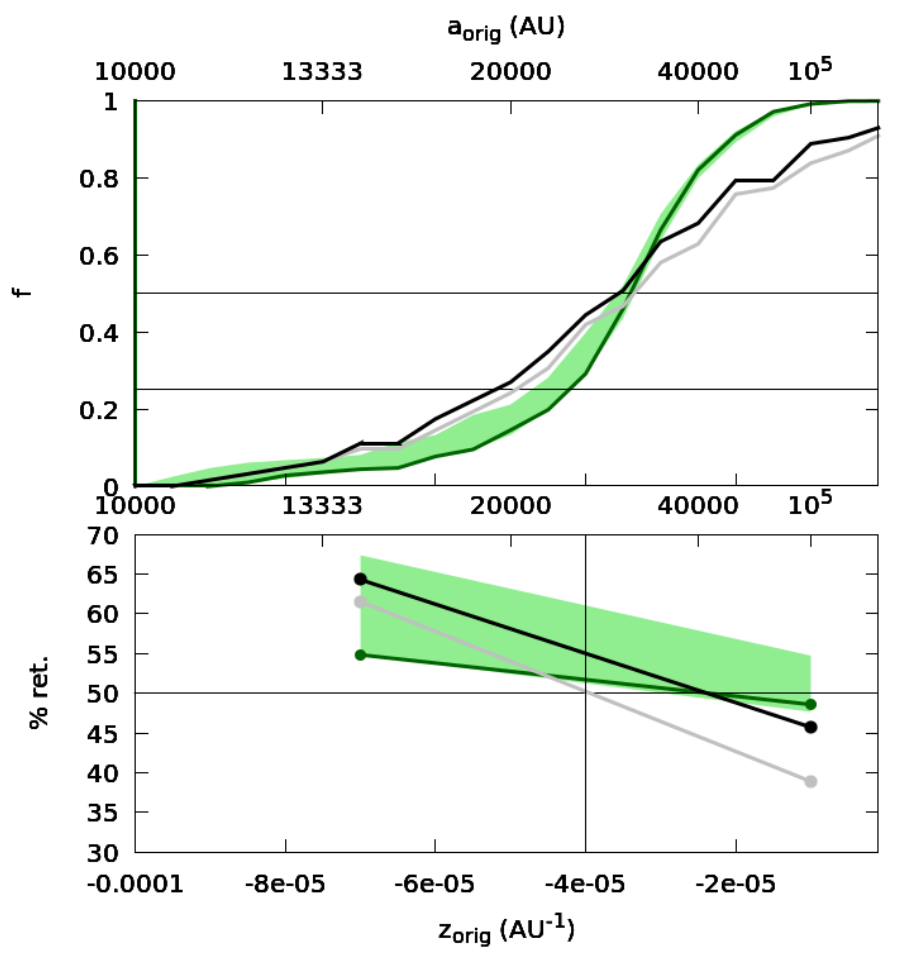

Fig. 5. Top panel: cumulative distribution of the original orbital energy for the observable new comets obtained from the simulations (green line), for the JPL $\mathrm{WC}_{\mathrm{WC}}$ sample (gray line), and for the WC sample (black line). Bottom panel: proportion of retrograde orbits for a semi-major axis smaller than $25000 \mathrm{AU}$ and for a semi-major axis greater than $25000 \mathrm{AU}$. The light green area corresponds to the range of results obtained from the ten simulations using different stellar sequences. The dark green line corresponds to the canonical sample shown in Fig. 4.

comets between our synthetics samples and the observations. Different hypotheses are proposed in the conclusions.

\subsubsection{Perihelion distance}

We now consider the distribution of perihelion distance. Figure 6 shows the density distribution and the cumulative distribution of the perihelion distance for the two observed samples and the reference simulation sample. Because the number of comets for the two observed samples is low, the distribution is very sensitive to the choice of the bin. The cumulative distribution is independent of this choice and shows that the agreement with the simulation sample is rather good, most of all for the JPL $\mathrm{WC}_{\mathrm{C}}$ sample. For the WC sample, an excess of comets is observed for a perihelion distance just above $1 \mathrm{AU}$. The reason might be that this sample is not complete and that observations between 1950 and 1998 were biased toward this perihelion distance (this can indeed be observed in Fig. 1).

When we us all simulations to estimate the range of the results on the cumulative distribution, we obtain Fig. 7. The figure confirms that the cumulative distribution obtained with the $\mathrm{JPL}_{\mathrm{WC}}$ catalog is consistent with the distribution obtained from the simulations.

Returning to the distribution of perihelion distance for the simulation sample, we note that the number of comets per perihelion distance unit increases with perihelion distance. This trend is also present in the observed samples, since their cumulative distribution remains below the cumulative distribution, which corresponds to a flat distribution, as is observed in Fig. 7. 


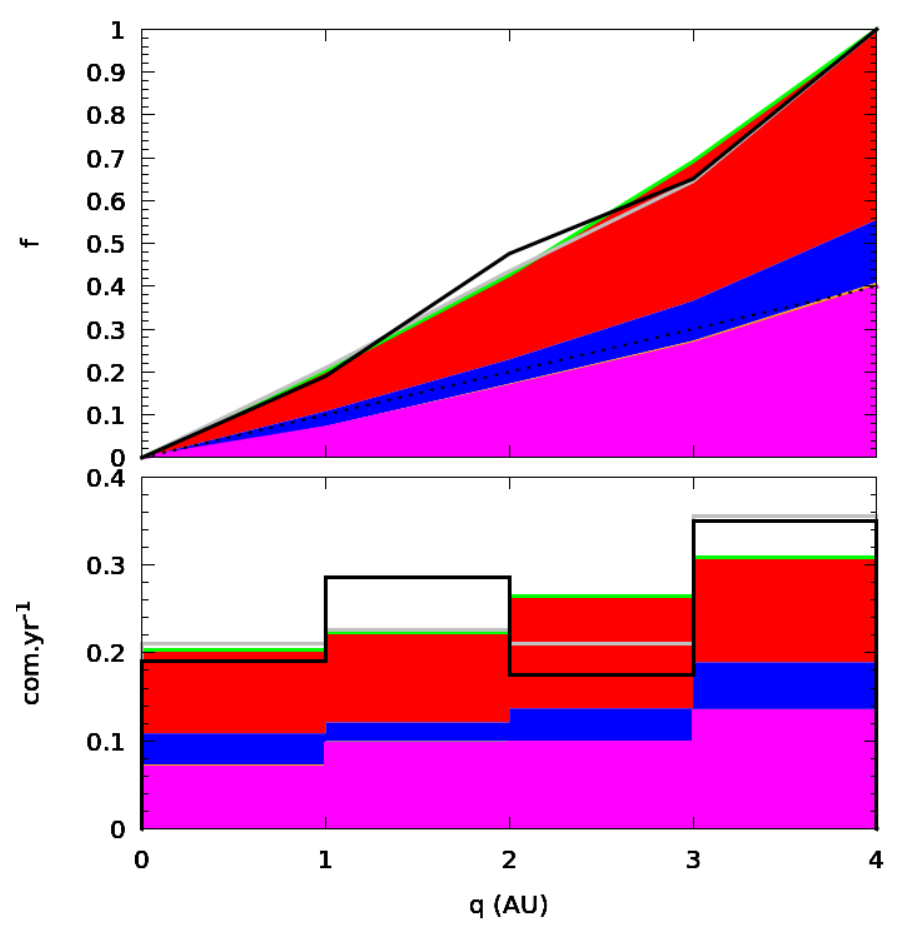

Fig. 6. Cumulative distribution (top pannel) and distribution (bottom panel) of the perihelion distance for the JPL $\mathrm{WC}_{\mathrm{WC}}$ sample (gray line), the WC sample (black line), and the simulation sample (green line). For the simulation sample, the colored area is proportional to the number of comets in each class using the same color code as for Fig. 4.

In Sect. 4.2 we increase the observable limit to $10 \mathrm{AU}$ and then investigate this trend.

From the simulation samples we see that this increase mainly stems from the increase in KQ-creepers, and to a smaller extent, from the creepers. Most of the jumpers come from the outer part of the Oort cloud (see Fig. 4), where the tides can easily decrease the perihelion distance to a very low value. In contrast, creepers and KQ-creepers have a lower semi-major axis, which means that the transport of the perihelion distance is less efficient than for jumpers, hence it is more difficult to penetrate deep inside the observable region before they are ejected.

\subsection{Observable comets with $q<10 \mathrm{AU}$}

We now extend the observable limit to 10 AU. The observed samples will clearly be incomplete for this perihelion distance, but a comparison with observations may be useful to evaluate the level of completeness. We can also hope that the discrepancy observed in Sect. 4.1 in the Oort spike will not affect the qualitative results of this section.

The new JPL $\mathrm{WC}_{\mathrm{WC}}$ sample now contains 117 comets, 8 of which are on hyperbolic orbits, and 72 have their original orbital energy taken from the Warsaw catalog (none of which are on hyperbolic orbits). The new WC catalog contains 112 comets, 5 of which are on hyperbolic orbits.

\subsubsection{Perihelion distances}

We first consider the distribution of perihelion distances. Figure 8 is the same as Fig. 6, this time for new comets with a perihelion distance smaller than $10 \mathrm{AU}$. The distributions have been normalized in a different way, however. The distribution

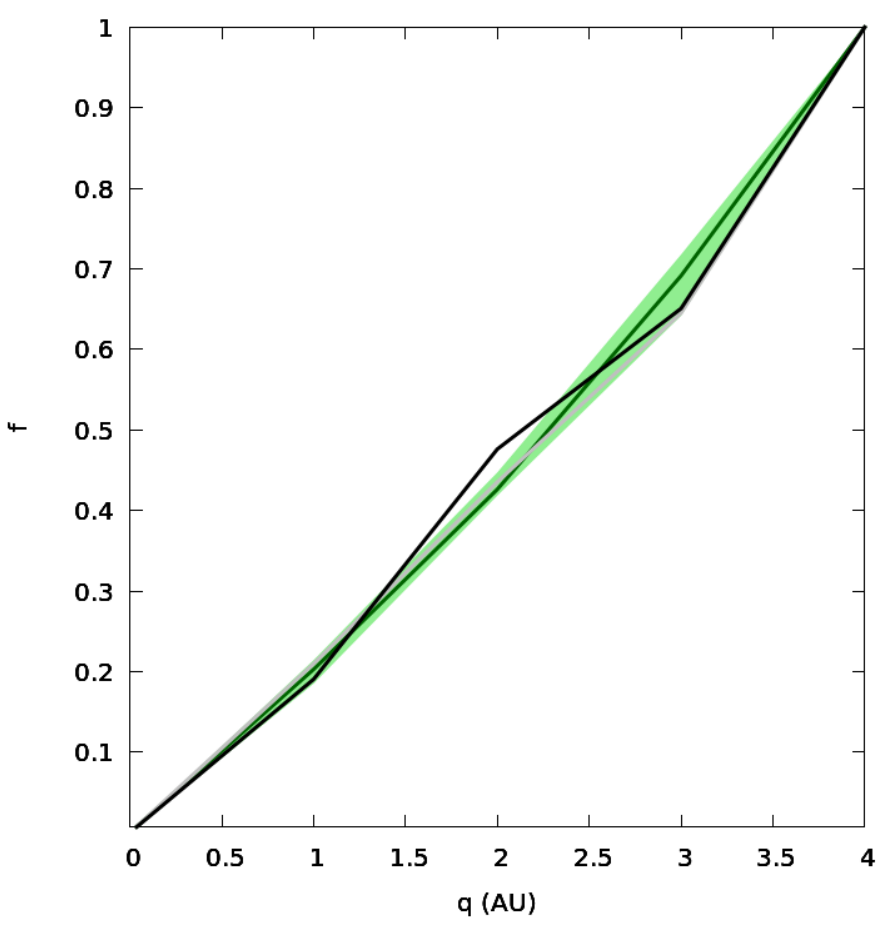

Fig. 7. Cumulative distribution of the perihelion distances for the JPL $L_{W C}$ sample (gray line), the WC sample (black line), and the simulation sample (green line). The light green area corresponds to the range of results obtained over our ten simulations. The dotted line corresponds to the cumulative distribution for a flat distribution of the perihelion distance.

itself has been normalized to comet/year for each sample (for the WC sample we considered the same normalization factor as for the JPL $L_{W C}$ sample since the total number of comets is approximately the same). For the cumulative distributions, the simulation sample was normalized as usual, and for the observed samples we considered that the flux was complete up to $q<4 \mathrm{AU}$, that is, for $q=4 \mathrm{AU}$, all three cumulative distributions have the same value. In this way, it is easy to visualize the incompleteness of the observed samples.

The observed samples are clearly incomplete beyond $4 \mathrm{AU}$. Of all comets with $q<10$ AU, only $35 \%$ have been observed and most of them have a perihelion distance smaller than $4 \mathrm{AU}$. For $q>7 \mathrm{AU}$, for instance, the observed flux per astronomical unit of perihelion distance is lower than 0.5 comet/year, whereas the simulation sample shows a flux greater than 3 comet/year.

We now examine the flux obtained from the simulation sample. This flux is well approximated by an exponential law with a number of comet per year and per perihelion distance unit $\alpha \mathrm{e}^{\alpha q}$ with $\alpha=0.201 \pm 0.007$. It is not possible to fit the distribution using a single power law over the entire range of perihelion distance, but when we separately consider comets with a perihelion distance smaller than $4 \mathrm{AU}$ and those with a perihelion distance between 6 and $10 \mathrm{AU}$, it is possible to fit the cumulative distribution by two different power laws. For $Q<4$ AU, we have $N(q<Q) \propto Q^{1.16}$, and for $4<Q<10 \mathrm{AU}$, we have $N(q<Q) \propto Q^{2.07}$. Figure 9 shows the cumulative distributions of the perihelion distance for observable comets in log-scale and the three different fits considered for our stellar sequence. The green area corresponds to the range of the results obtained over the ten different simulation using different stellar sequences. The knee between 4 and $6 \mathrm{AU}$ is well observed. Of the ten simulations, the first power law, that is, the power law for perihelion 


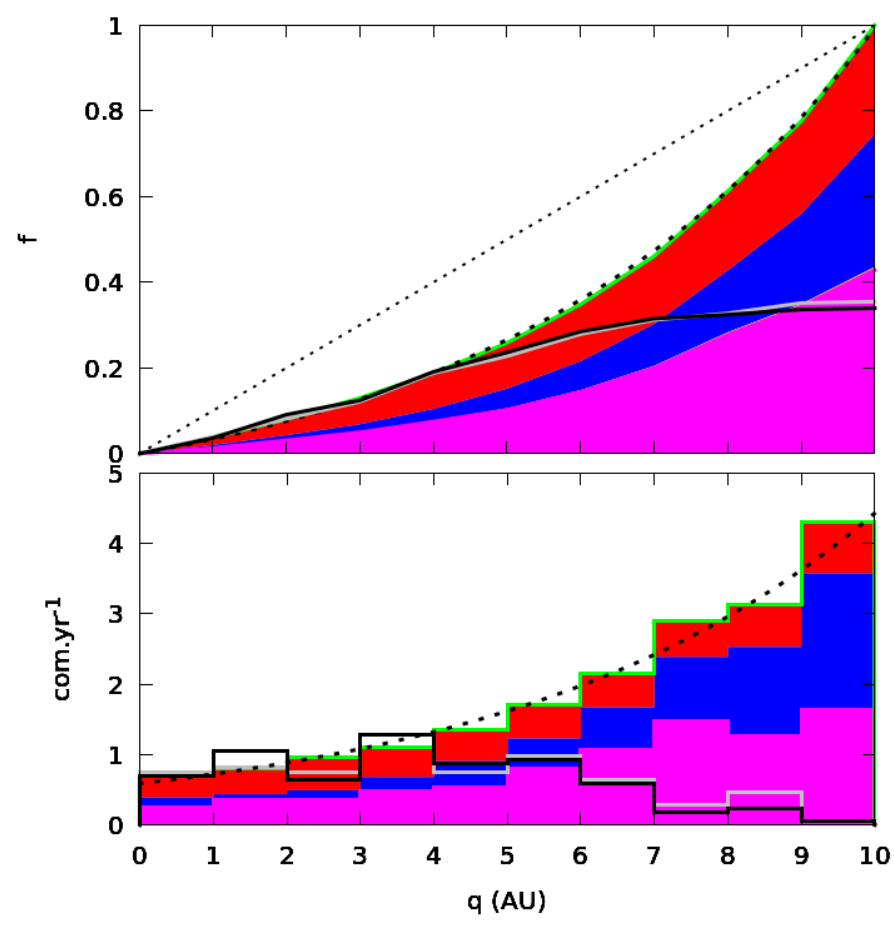

Fig. 8. Same as Fig. 6 considering new comets with a perihelion distance smaller than $10 \mathrm{AU}$. In addition, the dashed black curve corresponds to the best exponential law fit to the simulation distribution. The distributions have been normalized in a different way as for Fig. 6 (see text for details)

distance smaller than $4 \mathrm{AU}$, has a power ranging between 1.04 and 1.16 with a mean value equal to 1.09 , and for a perihelion distance between 6 and $10 \mathrm{AU}$, the power ranges between 2.06 and 2.33 with a mean value equal to 2.13 . For a fully thermalized Oort cloud we would expect that the number of comets with perihelion distance smaller than $q$ is proportional to $q^{2}$ (Hills 1981), and might be close to a linear increase for a small perihelion distance. Our results are in accordance with this statement; the discrepancy is probably caused by the fact that our Oort cloud is not fully thermalized, as has been observed in Fouchard et al. (2017).

The flux of new comets with $q<4$ AU is 165.9 comet/Myr, with $44 \%$ of jumpers ( 73.3 comet/Myr), $1 \%$ of KQ-jumpers (1.7 comet/Myr), $15 \%$ of creepers (24.1 comet/Myr) and $40 \%$ of KQ-creepers (65.7 comet/Myr). For $q<10 \mathrm{AU}$, the flux is 877.3 comet/Myr, with $25 \%$ of jumpers (222.8 comet/Myr), $1 \%$ of KQ-jumpers (6.9 comet/Myr), $31 \%$ of creepers (272.1 comet/Myr) and $43 \%$ of KQ-creepers (375.5 comet/Myr). Clearly, the increase in flux of comets with perihelion distance between 4 and 10 AU mainly comes from the contribution of the creepers and KQ-creepers. It is even more evident considering the increase in proportion to the flux for $q<4 \mathrm{AU}$ : for the total number of comets, the increase is about $430 \%$. A flat distribution of the perihelion distance for the flux of new comets would have yielded an increase of $150 \%$. For the four different observable classes the increase is about $200 \%, 300 \%, 1000 \%$, and $470 \%$ for the jumpers, KQ-jumpers, creepers, and KQ-creepers groups, respectively.

Because the number of KQ-jumpers is very small, their increase is not significant here. For the jumpers, we recall that for a comet to be a jumper, its perihelion distance had to be larger than $15 \mathrm{AU}$ at the perihelion one orbital period before it is "observed".

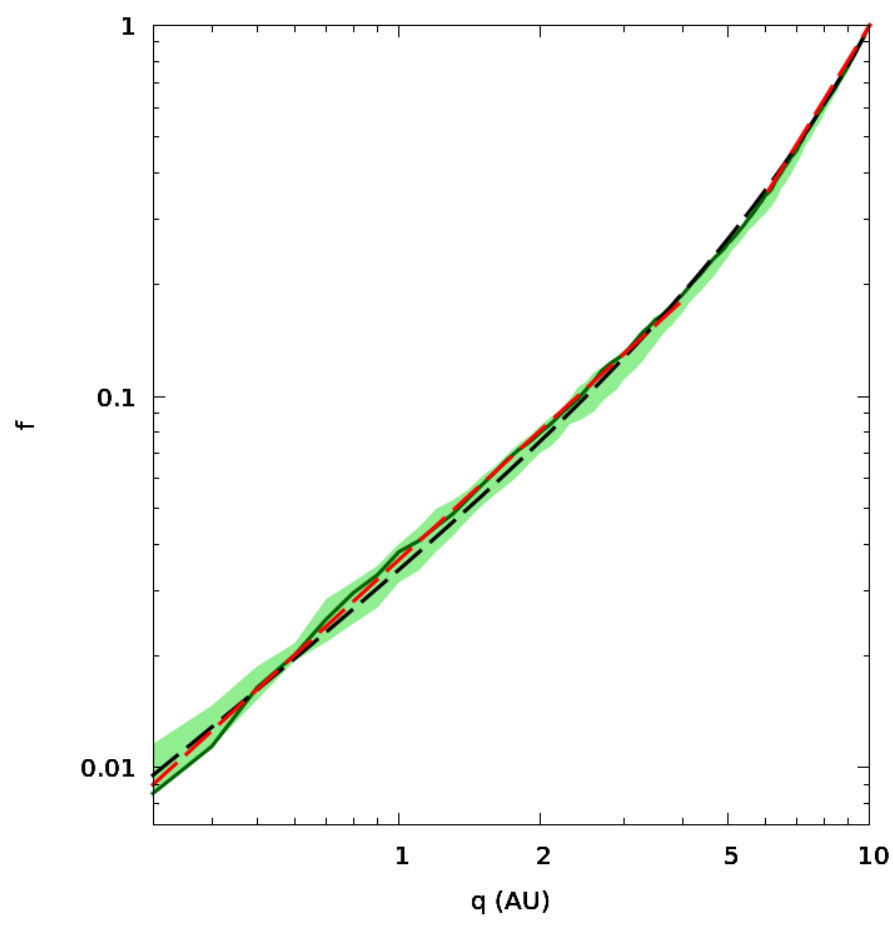

Fig. 9. Cumulative distribution of the perihelion distances for the JPL ${ }_{W C}$ sample (gray line), the WC sample (black line), and the simulation sample (green line). The light green area corresponds to the range of results obtained over our ten simulations. The dotted line corresponds to the cumulative distribution for a flat distribution of the perihelion distance.

Consequently, increasing the observable limit from 4 to $10 \mathrm{AU}$ facilitates the production of jumpers. Surprisingly, the increase is rather moderate ( $200 \%$ instead of $150 \%$ for a flat distribution). This contributes to the conclusion reached in Sect. 4.1, that is, because the jumpers are the comets with the largest semi-major axis, the tides have a similar efficiency in decreasing the perihelion distance below 10 or $4 \mathrm{AU}$

In contrast, the number of creepers has drastically increased. We know from Fouchard et al. (2014) that most of the creepers are fast creepers, that is, they have spent only one perihelion passage closer than 15 AU before they were observed. When the observable limit is increased, we observe creepers that would have needed to spend more than one perihelion passage closer than $15 \mathrm{AU}$ to reach a heliocentric distance smaller than $4 \mathrm{AU}$, but they have been ejected by Jupiter or Saturn before this. By increasing the limit of the observable zone, we are now able to observe these creepers probably at the perihelion where they are ejected from the Oort cloud.

In addition, according to Fouchard et al. (2013), the probability of ejection (or opacity factor) between 10 and 15 AU is about $30 \%$, whereas it is about $80 \%$ between 5 and $10 \mathrm{AU}$. Hence, a typical potential creeper comet when the observable limit for the perihelion distance is equal to $10 \mathrm{AU}$ has its previous perihelion distance between 10 and $15 \mathrm{AU}$, with a moderate chance to be ejected by planetary scattering, whereas when the observable limit is equal to $4 \mathrm{AU}$, a typical potential creeper has its previous perihelion distance between 4 and $15 \mathrm{AU}$, with a much higher probability of ejection by planetary scattering. The drastic change in the opacity factor in this region very well explains the huge increase in creepers observed here between an observable limit at $4 \mathrm{AU}$ and an observable limit at $10 \mathrm{AU}$. 


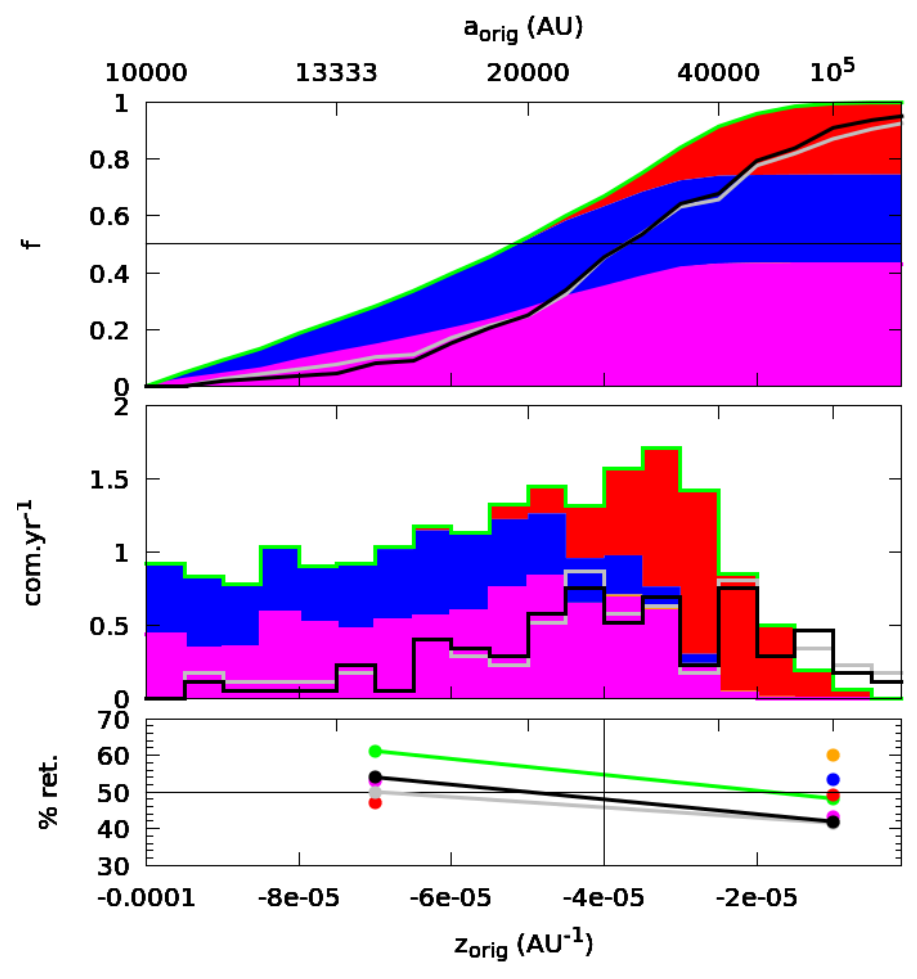

Fig. 10. Same as Fig. 4 considering new comets with a perihelion distance smaller than $10 \mathrm{AU}$.

For the KQ-creepers, the increase is less pronounced, and we see in Fig. 8 that the increase in KQ-creepers shows a knee at about 7-8 AU. This occurs because the comets feel the influence of Jupiter much less strongly beyond this limit, hence it is harder for a comet to receive the planetary kick that makes it a KQ-comet. On the other hand, as we observed above, this favors the production of creepers. We clearly see in the bottom plot of Fig. 8 that the lack of KQ-creepers beyond 7-8 AU corresponds to the gain of creepers beyond this limit.

\subsubsection{Orbital energy and inclination}

Figure 10 is the same as Fig. 4, this time for new comets with a perihelion distance smaller than $10 \mathrm{AU}$. As for Fig. 8, in the bottom plot the data are normalized in number of comets per year.

The shape of the spike between simulation and observations is now drastically different. The first quartile and the median of the original orbital energy is $20200 \mathrm{AU}$ and $27400 \mathrm{AU}$ for the WC sample and 20800 AU and 27300 AU for the JPL ${ }_{W C}$ sample, whereas these quantities are $13500 \mathrm{AU}$ and $19400 \mathrm{AU}$ for the simulation sample, respectively. The inner part of the spike, where $a_{\text {orig }}<20000 \mathrm{AU}$, is missing in the observed samples. This discrepancy is easily explained because the missing comets in the observed samples are comets with higher perihelion distance, and these comets are mainly creepers and KQ-creepers. As we recalled before, these comets mainly come from regions of the Oort cloud where $a<20000$ AU.

The proportion of retrograde comets shown in the bottom panel of Fig. 10 increases for comets with a semi-major axis smaller than $25000 \mathrm{AU}$ for the simulation sample. This is most likely due to the even larger proportion of creepers and KQcreepers, and we know from Fouchard et al. (2014) that they prefer retrograde orbits to increase their chance of reaching the observable region.

\section{Conclusions}

We have performed a comparison between the observed flux of new long-period comets and a synthetic flux obtained from a model that is assumed to reproduce the same dynamical environment as the present solar system (see Fouchard et al. 2017, for more details). Ten different synthetic fluxes were obtained, considering different sequences of stellar encounters with the solar system. Two different thresholds for the maximum heliocentric distance were considered for a comet to be observable. At the first threshold at $4 \mathrm{AU}$, one of our observed samples can be considered complete for comets with a total magnitude lower than 11. The second threshold at $10 \mathrm{AU}$ was considered to evaluate the incompleteness of the observed samples and the characteristics of the flux of comets beyond $4 \mathrm{AU}$.

For the threshold at $4 \mathrm{AU}$, some small but significant discrepancies were observed between the synthetic samples and the observed samples. In particular, the first quartile of the original orbital energy for the comets in the Oort spike is higher for the synthetic samples than for the observed samples, and the proportion of retrograde comets for $a_{\text {orig }}>25000 \mathrm{AU}$ is around $50 \%$ for our synthetics samples, whereas as it is $54 \%$ or higher for our observed samples.

It is difficult to draw a conclusion from these discrepancies. However, some hypotheses can be proposed.

(i) The determination of the original orbital energy of known long-period comets can be further improved and it may change the results, especially the proportion of retrograde orbits.

(ii) We have shown in Fouchard et al. (2011) that stellar perturbations during the last orbital period increase the chance for comets with moderate original orbital energy to become observable. Hence the first quartile and the median of the original orbital energy of the comets in the Oort spike are smaller than in the case where no stellar perturbation are present during the last orbital period of the comets. Consequently, the discrepancy between simulations and observations observed in the present paper might be an indication that the present frequency of stellar encounters is higher than the frequency considered in our simulations. However, this may mainly concern very low-mass stars that are not able to disrupt the Oort cloud or produce comet showers. These unseen stars are expected only to affect the Oort cloud comets in the close neighborhood of their trajectory.

(iii) Batygin \& Brown (2016) have proposed the existence of a massive planet between the extended scattered disk and the Oort cloud. The effect of such a planet on the flux of longperiod comets has not yet been studied. It is be possible that our discrepancy comes from such effects.

(iv) The initial conditions should clearly have an effect on the final characteristics of the Oort spike.

Points (ii), (iii), and (iv) will be the subject of a forthcoming paper. 
For the threshold at $10 \mathrm{AU}$ our conclusions are as follows.

(i) The synthetic samples show that the number of observable comets per year and per perihelion distance unit is $\propto q^{1.09}$ for $q<4 \mathrm{AU}$ and $\propto q^{2.13}$ for $6<q<10 \mathrm{AU}$. About $65 \%$ of the comets are still unobserved.

(ii) The exponential increase of comets mainly stems from KQ-creepers for perihelion distances smaller than 7-8 AU, and creepers beyond this limit.

(iii) These comets mainly come from the inner part of the Oort spike, that is, have semi-major axes smaller than $20000 \mathrm{AU}$.

A deep investigation of the observed Oort spike and new simulations considering different dynamical scenarios for the evolution of our synthetic comets should help in our understanding of the dynamical history of the Oort cloud and will be the scope of a forthcoming paper. Observations of comets beyond $5 \mathrm{AU}$ would be important in this respect, mainly because these comets would be coming from semi-major axes smaller than $20000 \mathrm{AU}$, where the Oort cloud could have kept some more memory of its initial shape.

Acknowledgements. We gratefully acknowledge the remarks made by the referee of this paper that greatly helped to improve the quality of the paper.

\section{References}

Batygin, K., \& Brown, M. E. 2016, AJ, 151, 22

Brasser, R., \& Morbidelli, A. 2013, Icarus, 225, 40

Dybczyński, P. A., \& Królikowska, M. 2011, MNRAS, 416, 51

Dybczyński, P. A., \& Królikowska, M. 2015, MNRAS, 448, 588

Fernández, J. A., \& Sosa, A. 2012, MNRAS, 423, 1674

Fouchard, M., Rickman, H., Froeschlé, Ch., \& Valsecchi, G. B. 2011, A\&A, 535, A86

Fouchard, M., Rickman, H., Froeschlé, Ch., \& Valsecchi, G. B. 2013, Icarus, 222, 20

Fouchard, M., Rickman, H., Froeschlé, Ch., \& Valsecchi, G. B. 2014, Icarus, 231,110

Fouchard, M., Rickman, H., Froeschlé, Ch., \& Valsecchi, G. B. 2017, Icarus Francis, P. J. 2005, ApJ, 635, 1348

Giorgini, J., Yeomans, D., Chamberlin, A., et al. 1996, BAAS, 28, 1158

Hills, J. G. 1981, AJ, 86, 1730

Kaib, N. A., \& Quinn, T. 2009, Science, 325, 1234

Królikowska, M. 2006, Acta Astron., 56, 385

Królikowska, M. 2014, A\&A, 567, A126

Levison, H. F., Dones, L., \& Duncan, M. J. 2001, AJ, 121, 2253

Levison, H. F., Duncan, M. J., Dones, L., \& Gladman, B. J. 2006, Icarus, 184 619

Rickman, H., Fouchard, M., Froeschlé, Ch., \& Valsecchi, G. B. 2008, Celes Mech. Dyn. Astron., 102, 111

Todorovic-Juchnicwicz, B. 1981, Acta Astron., 31, 191

Wiegert, P., \& Tremaine, S. 1999, Icarus, 137, 84 